\title{
Reliability and validity of a short FFQ for assessing the dietary habits of 2-5-year-old children, Sydney, Australia
}

\author{
Victoria M Flood ${ }^{1, *}, t$, Li Min Wen ${ }^{2,3}$, Louise L Hardy ${ }^{3}$, Chris Rissel ${ }^{2,3}$, Judy M Simpson ${ }^{3}$ \\ and Louise A Baur ${ }^{3,4}$ \\ 'School of Health Sciences, Faculty of Health and Behavioural Sciences, University of Wollongong, NSW 2455, \\ Australia: ${ }^{2}$ Health Promotion Service, Sydney South West Area Health Service, Sydney, Australia: ${ }^{3}$ School of \\ Public Health, University of Sydney, Sydney, Australia: ${ }^{4}$ Discipline of Paediatrics \& Child Health, University of \\ Sydney, Sydney, Australia
}

Submitted 27 January 2012: Final revision received 4 January 2013: Accepted 22 January 2013: First published online 1 May 2013

\begin{abstract}
Objective: A simple FFQ which ranks young children's dietary habits is necessary for population-based monitoring and intervention programmes. The aim of the present study was to determine the reliability and validity of a short FFQ to assess the dietary habits of young children aged 2-5 years.

Design: Parents completed a seventeen-item FFQ for their children by telephone on two occasions, two weeks apart. Sixty-four parents also completed $3 \mathrm{~d}$ food records for their children. The FFQ included daily servings of fruit and vegetables, frequency of eating lean meat, processed meats, take-away food, snack foods (biscuits, cakes, doughnuts, muesli bars), potato crisps and confectionery, and cups of soft drinks/cordials, juice, milk and water. Weighted kappa and intra-class correlation coefficients were used to assess FFQ reliability and the Bland-Altman method was used to assess validity of the FFQ compared with the $3 \mathrm{~d}$ food record. Setting: Seven pre-school centres in metropolitan Sydney, Australia.

Subjects: Seventy-seven children aged 2-5 years.

Results: The majority of questions had moderate to good reliability: $\kappa_{\mathrm{w}}$ ranged from 0.37 (lean meat) to $0 \cdot 85$ (take-away food consumption). Validity analysis showed a significant increase in mean values from the food record with increasing ordered categories from the FFQ for servings of vegetables and fruit and cups of drinks (all trend $P \leq 0 \cdot 01$ ). Spearman rank correlation coefficient was $>0.5$ for vegetables, fruit, diet soft drinks and fruit juice.

Conclusions: The FFQ provides reliable and moderately valid information about the dietary intakes and habits of children aged 2-5 years, in particular for fruit, vegetables and beverages.
\end{abstract}

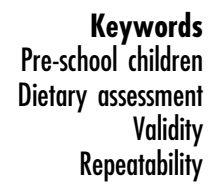

Population-based surveys frequently use short questions to assess lifestyle habits in order to identify patterns and habits quickly and easily, provide information that can be used to monitor the population over time, and identify potential lifestyle habits requiring intervention. In assessing dietary intake, various FFQ have been developed and validated for use in general adult and older child populations ${ }^{(1-4)}$; however, few short FFQ have been developed to assess the dietary habits of children of pre-school age.

Reliability and validity studies of FFQ have been conducted in pre-school children in Belgium and Canada to determine healthy eating scores ${ }^{(5,6)}$. In the Belgian study, an FFQ with forty-seven items was used to assess

$\dagger$ This research was partially conducted while the first author was employed at the University of Sydney. usual nutrient and food intakes over the past year for children aged 2-6 years and compared with food and nutrient intakes obtained using a $3 \mathrm{~d}$ food record $(n 650)^{(6)}$. In Canada, the NutriSTEP ${ }^{\circledR}$ (Nutritional Screening Tool for Every Preschooler) is a seventeen-item questionnaire with five questions focusing on food groups and the remainder on nutritional risk constructs such as physical growth, food and fluid intake, and physical activity among others, and it was used to derive an index ${ }^{(5)}$. The NutriSTEP was tested for criterion validity and test-retest reliability against a $3 \mathrm{~d}$ dietary recall and was found to be valid (in terms of a score to define nutritional risk) and repeatable ( $\kappa$ ranging from $>0.5$ to $>0.75$ on most items; intra-class correlation coefficient (ICC) $=0 \cdot 89$ ). In another European study, the repeatability of a forty-three-item FFQ was assessed among 2-9-year-old children ( $n$ 258), but no assessment of validity was conducted $^{(7)}$. 
In Australia, there have been a few reliability and validation studies conducted in older children of primaryschool age ${ }^{(3,4)}$ but little research among populations of pre-school children. There was one Australian study which validated a twenty-eight-item diet survey among 4-16-year-old Australian children in which a longer $7 \mathrm{~d}$ food checklist was used to assess agreement, and this was subsequently used for a scoring system ${ }^{(8)}$. International researchers have also conducted validity studies of shorter tools among school-aged children, including Baranowski et $a l^{(9)}$, Prochaska and Sallis ${ }^{(10)}$, Rockett et al. ${ }^{(11)}$, Vereecken et $a l^{(12)}$ and Lilliegaard ${ }^{(13)}$, but these are limited in age range (3rd grade students, ages 12 years, 9-14 years, 11-12 years, and 9 and 13 years, respectively). This probably reflects the difficulty of obtaining accurate dietary data from young children, as it needs to be obtained by proxy and attention needs to be given to alternative carers, such as child-care services who may be providing food to the child outside the home ${ }^{(14)}$.

In summary, to date there has been no validity or repeatability assessment of short questions among children aged $2-5$ years in Australia and very few have occurred internationally ${ }^{(5-7,15)}$. The purpose of the present study was to determine the reliability and validity of a seventeen-item FFQ which assessed the dietary intake and habits of children aged $2-5$ years, as reported by their parents and pre-school carers.

\section{Methods}

\section{Design}

The present cross-sectional study of parents of pre-school children comprised a convenience sample of pre-school children, collected between September 2007 and April 2008. The study was approved by the University of Sydney Human Research Ethics Committee.

\section{Participants}

Ten pre-school centres located in metropolitan Sydney, NSW, Australia were approached to participate in the study and seven agreed. Within each of these seven pre-school centres, parents of children aged 2-5 years were invited to participate. Informed consent by parents was a requirement for participation.

\section{Short FFQ}

A seventeen-item FFQ was developed, based on previous questions commonly used in the NSW Government's Population Health Survey ${ }^{(16,17)}$. The questions related to food and beverage items that are associated with health, designed to assess usual intake, and included daily servings of fruit and vegetables, usual frequency of eating lean meat, processed meats, take-away food, snack foods (biscuits, cakes, doughnuts, muesli bars), potato crisps and confectionery, and cups of sugary drinks (soft drinks, cordials, and sports drinks), fruit juice, milk and water, frequency of eating in front of the television and requency of daily breakfast consumption (see Appendix). For the reliability study the short FFQ was administered by telephone on two occasions within a fortnight of each other.

\section{Diet records}

For the validity study, parents were asked to complete $3 \mathrm{~d}$ food records (FR) within two weeks after the administration of the second FFQ. Parents were contacted by research staff and provided with household measures and instructions on how to record everything their child ate or drank on two weekdays and one weekend day. Pre-school staff were also provided with instructions on how to complete the FR if the participating child was at pre-school on one or both of the weekdays.

\section{Otber measures}

Parents of the children were asked to provide general demographic characteristics, including the child's sex, date of birth and postcode of residence. Two researchers visited each pre-school and measured the height $(\mathrm{m})$ and weight (kg) of each child, using an electronic scale and portable stadiometer. These data were used to assess the plausibility of the reported energy intake data from the $3 \mathrm{~d}$ FR.

\section{Statistical analysis}

Data were analysed using the SPSS statistical software package version $15 \cdot 0$. The data from the FFQ were analysed using both continuous and categorical methods, because the data may be used in either way; for example, in population health monitoring, data from short FFQ are frequently reported in terms of those meeting recommended servings for fruit and vegetables ${ }^{(18)}$. For the reliability study, the short FFQ responses were categorised according to the distribution and frequency of responses. The proportion of parents reporting within the same response category, between surveys, was determined and weighted kappa values $\left(\boldsymbol{\kappa}_{\mathrm{w}}\right)$ were calculated using MedCalc statistical software version $10 \cdot 0 \cdot 1 \cdot 0$. The $\kappa_{\mathrm{w}}$ values were characterised as showing poor agreement $(<0 \cdot 20)$, fair agreement $(0 \cdot 20-0 \cdot 40)$, moderate agreement $(0 \cdot 41-0 \cdot 60)$, good agreement $(0 \cdot 61-0 \cdot 80)$ and very good agreement $(0 \cdot 81-1 \cdot 00)^{(19)}$. Agreement between FFQ responses was also determined for the raw data using the ICC statistic, as described in Armitage et al. ${ }^{(20)}$.

In order to conduct the concurrent validation of the seventeen-item FFQ, the $3 \mathrm{~d}$ FR were entered into the dietary software analysis package Foodworks Professional version 5 and the AUSNUT 1999 nutrient database ${ }^{(21)}$ was used for analysis. The average weight $(\mathrm{g})$ of the food items from the FR was compared with the servings, frequency or cups reported in the first short FFQ. To calculate servings of fruit, the weight of all fruit was divided by 
$150 \mathrm{~g}$, and to calculate servings of vegetables the weight of all vegetables was divided by $75 \mathrm{~g}$, as used in the Australian Guide to Healthy Eating and reported in previous analyses of servings of fruit and vegetables ${ }^{(22)}$. This measure of concurrent validity assessed food items reported in the short FFQ compared with the more comprehensive FR, sometimes referred to as 'direct' validity in dietary data analysis ${ }^{(23)}$. Additionally, indirect validity was investigated by examining selected nutrients for each short question. 'Indirect' validity compares the food items reported in the FFQ with the nutrient intake derived from the FR, similar to the method reported by the Australian Food and Nutrition Monitoring Unit ${ }^{(24)}$. For example, given the known vitamin $\mathrm{C}$ content of juice and fruit, the total vitamin $\mathrm{C}$ intake of the diet is evaluated in relation to categories of responses for fruit and juice, providing an indication of indirect validity. The plausibility of the energy intake from the $3 \mathrm{~d}$ FR was determined using the Goldberg cut-offs for energy intake for a physical activity level of 1.60 ( $\pm 95 \%$ confidence limits), as described by Black ${ }^{(25)}$. The food items from the FFQ were categorised into quartiles, to the nearest half or whole serving, frequency or cups, as applicable. Since many people may provide the same response (e.g. one serving daily, zero soft drinks), the numbers were not always distributed evenly across the four categories.

In addition, the validity of the data was assessed using the continuous data, comparing the servings, frequency or cups of food reported in the FFQ with the FR, using both a paired $t$ test and the Spearman rank correlation coefficient. Using the FR as the criterion standard, we also estimated the sensitivity, specificity, positive predictive value and negative predictive value (with exact binomial confidence intervals) of the FFQ for the items of fruit and vegetables in relation to recommended servings (one serving or more for fruit, two or more servings for vegetables), as this comparison is frequently reported in state and national nutrition monitoring ${ }^{(18,26)}$.

Additionally, the average servings of fruit and vegetables and cups of milk and water were assessed using the Bland-Altman method ${ }^{(27)}$, where the mean of the two measurement methods ((first FFQ + FR)/2) was plotted on the $x$-axis and the difference (first FFQ $-\mathrm{FR}$ ) was plotted on the $y$-axis. Any systematic difference between the two methods was also assessed using a regression test.

Three questions from the FFQ were not included in the validity assessment (breakfast consumption, take-away use and eating in front of the television) as these items were not consistently reported in the FR by parents.

\section{Results}

Seventy-seven parents of children aged 2-5 years participated in the reliability study and sixty-four parents participated in the validity study. The majority of parents interviewed were mothers (96\%) aged 25-45 years (92\%), with a tertiary education (90\%) and from Englishspeaking backgrounds (93\%). The mean age of the children was 3.6 (SD 0.94) years. The mean age and age range of children and their parents did not differ between those who participated in both the repeatability and validity studies; however, there were fewer parents with high school only education among the parents who provided the validity data $(3 \% v .10 \%$ in FR and FFQ, respectively).

\section{Reliability}

Table 1 reports the proportion within each response category and the $\kappa_{\mathrm{w}}$ values for each FFQ item. The majority of items showed moderate to good agreement with $\kappa_{\mathrm{w}}$ values ranging from 0.37 for red meat frequency to $0 \cdot 85$ for take-away food frequency. Similarly, the ICC for the raw data showed moderate to good agreement and ranged from $0 \cdot 29$ for red meat frequency to $0 \cdot 82$ for salty snack food frequency. Overall, eleven of the seventeen items showed good (fruit, hot potato chips, take-away food, eating in front of the television, milk, regular and diet soft drink, fruit juice and water) to very good (salty snacks, confectionery) agreement.

\section{Validity}

The concurrent validity of the short questionnaire is shown in Tables 2 (foods) and 3 (drinks), where the mean and 95\% confidence interval of the FR data are shown for a range of items, classified by FFQ category, based on responses to the first FFQ. The servings of fruit and vegetables from the FR indicated relatively good validity for servings reported by categories of the short FFQ, with $P$ for trend $<0 \cdot 001$ and mean servings similar to those described in the short questions. For example, those who reported consuming more than two servings of vegetables daily (FFQ) actually consumed a mean of 1.8 servings/d (FR), while those who reported consuming four or fewer servings of vegetables weekly actually consumed 0.7 servings/d (equivalent to 4.9 servings/week). There was a significant trend for increasing vitamin C intake among those reporting more servings of vegetables ( $P$ for trend $=0.03$ ) and a significant trend for an increasing fibre intake among those reporting more servings of fruit ( $P$ for trend $=0 \cdot 01$ ). There was no significant trend of frequency or weight for short questions on red meat, processed meat, hot chips, potato crisps and snack foods. There was a significant trend for increasing weight of confectionery ( $P$ for trend $=0 \cdot 04$ ), with those reporting four or more servings of confectionery weekly (FFQ) consuming a mean of $6.6 \mathrm{~g} / \mathrm{d}$ (FR), compared with a mean of $2.7 \mathrm{~g} / \mathrm{d}$ among those who reported consuming confectionery less than once weekly.

There were significant increasing trends for indirect measures of validity in the following items: energy $(P=0 \cdot 02)$ and protein intake $(P=0.05)$ with increasing 
Table 1 Repeatability of the short FFQ among pre-school children ( $n$ 77) aged 2-5 years, metropolitan Sydney, Australia, September 2007-April 2008

\begin{tabular}{|c|c|c|c|c|c|}
\hline Food items & $\begin{array}{l}\text { Response } \\
\text { categories }\end{array}$ & $\begin{array}{l}\text { FFQ1, FFQ2 (\% within each } \\
\text { response category) }\end{array}$ & $\begin{array}{l}\text { Percentage } \\
\text { agreementt }\end{array}$ & $\kappa_{\mathrm{w}} \ddagger$ & ICC \\
\hline Vegetables (servings) & $\begin{array}{l}\leq 4 / \text { week } \\
5-7 / \text { week } \\
>1-2 / d \\
>2 / d\end{array}$ & $\begin{array}{l}16,12 \\
39,28 \\
33,47 \\
12,13\end{array}$ & 60 & 0.50 & 0.53 \\
\hline Fruit (servings) & $\begin{array}{l}\leq 1 / d \\
>1-2 / d \\
>2-3 / d \\
>3 / d\end{array}$ & $\begin{array}{l}25,24 \\
37,31 \\
25,37 \\
12,8\end{array}$ & 60 & 0.60 & $0 \cdot 78$ \\
\hline Red meat (frequency) & $\begin{array}{l}\leq 1 / \text { week } \\
>1-2 / \text { week } \\
>2-3 / \text { week } \\
>3 / \text { week }\end{array}$ & $\begin{array}{l}21,17 \\
15,25 \\
31,31 \\
33,27\end{array}$ & 53 & 0.37 & $0 \cdot 29$ \\
\hline Processed meat (frequency) & $\begin{array}{l}\leq 2 / \text { month } \\
\text { 1/week } \\
\text { 2-3/week } \\
>3 / \text { week }\end{array}$ & $\begin{array}{l}29,31 \\
23,29 \\
24,20 \\
24,20\end{array}$ & 60 & 0.47 & $0 \cdot 40$ \\
\hline Hot potato chips (frequency) & $\begin{array}{l}<1 / \text { month } \\
1 / \text { month } \\
2-3 / \text { month } \\
\geq 1 / \text { week }\end{array}$ & $\begin{array}{l}21,25 \\
23,27 \\
19,15 \\
37,33\end{array}$ & 69 & 0.69 & $0 \cdot 80$ \\
\hline Salty snacks (frequency) & $\begin{array}{l}<1 / \text { month } \\
1 / \text { month } \\
2-3 / \text { month } \\
\geq 1 / \text { week }\end{array}$ & $\begin{array}{l}29,24 \\
21,32 \\
15,13 \\
35,31\end{array}$ & 64 & 0.64 & $0 \cdot 82$ \\
\hline Take-away food (frequency) & $\begin{array}{l}<1 / \text { week } \\
1 / \text { week } \\
>1 / \text { week }\end{array}$ & $\begin{array}{c}79,79 \\
16,18 \\
4,3\end{array}$ & 86 & 0.85 & 0.77 \\
\hline Snack foods (biscuits) (frequency) & $\begin{array}{l}<1 / \text { week } \\
1-2 / \text { week } \\
>2-6 / \text { week } \\
\geq 1 / \text { d }\end{array}$ & $\begin{array}{l}16,12 \\
25,23 \\
21,31 \\
37,35\end{array}$ & 64 & 0.63 & 0.52 \\
\hline Confectionery (frequency) & $\begin{array}{l}<1 / \text { week } \\
1-1 \cdot 5 / \text { week } \\
2-3 / \text { week } \\
>3 / \text { week }\end{array}$ & $\begin{array}{l}32,27 \\
23,23 \\
20,35 \\
25,16\end{array}$ & 60 & 0.63 & $0 \cdot 83$ \\
\hline Breakfast (frequency) & $\begin{array}{l}\text { 5-6/week } \\
\text { Daily }\end{array}$ & $\begin{array}{c}4,2 \\
96,98\end{array}$ & 97 & 0.50 & $0 \cdot 30$ \\
\hline Dinner in front of television (frequency) & $\begin{array}{l}\leq 1 / \text { week } \\
>1 / \text { week }\end{array}$ & $\begin{array}{l}77,74 \\
23,26\end{array}$ & 86 & 0.63 & $0 \cdot 72$ \\
\hline Milk (cups; $250 \mathrm{ml}$ ) & $\begin{array}{l}<1 / d \\
1-1 \cdot 5 / d \\
2 / d \\
\geq 2 \cdot 5 / d\end{array}$ & $\begin{array}{l}13,7 \\
28,33 \\
31,35 \\
28,25\end{array}$ & 59 & 0.60 & $0 \cdot 74$ \\
\hline Soft drinks $\S$ (cups; $250 \mathrm{ml}$ ) & $\begin{array}{l}\text { Does not drink } \\
<1 / \text { week } \\
1-3 / \text { week } \\
\geq 3 \cdot 5 / \text { week }\end{array}$ & $\begin{array}{c}64,67 \\
13,12 \\
16,12 \\
7,9\end{array}$ & 72 & 0.38 & $0 \cdot 61$ \\
\hline Diet soft drinks (cups; $250 \mathrm{ml}$ ) & $\begin{array}{l}\text { Does not drink } \\
<1 / \text { week } \\
1-3 / \text { week } \\
\geq 3 \cdot 5 / \text { week }\end{array}$ & $\begin{array}{l}93,88 \\
1,5 \\
4,4 \\
1,3\end{array}$ & 91 & 0.65 & $0 \cdot 80$ \\
\hline Fruit juice (cups; 250 ml) & $\begin{array}{l}\leq 1 / \text { month } \\
>1 / \text { month to } 3 / \text { week } \\
0 \cdot 5-1 / \text { d } \\
\geq 1 \cdot 5 / d\end{array}$ & $\begin{array}{l}28,33 \\
37,28 \\
23,29 \\
12,9\end{array}$ & 68 & 0.69 & $0 \cdot 78$ \\
\hline Water (cups; $250 \mathrm{ml}$ ) & $\begin{array}{l}\leq 1 \cdot 5 / d \\
2-2 \cdot 5 / d \\
3-3 \cdot 5 / d \\
\geq 4 / d\end{array}$ & $\begin{array}{l}23,21 \\
27,31 \\
23,28 \\
28,20\end{array}$ & 63 & 0.58 & $0 \cdot 73$ \\
\hline
\end{tabular}

†Percentage within same category.

$\ddagger$ Weighted $\kappa\left(\kappa_{\mathrm{w}}\right)$ was calculated for categorised data and intra-class correlation coefficient (ICC) was calculated for raw data

§Includes soft drinks, syrups and sports drinks.

frequency of processed meat consumption; and mean sugars consumption with increasing snack food consumption $(P=0 \cdot 01)$.
Drinks tended to have good validity with means increasing as the reported cups consumed increased (Table 3). All drinks (milk, sugary drinks, fruit juices and 
Table 2 Relative validity: mean and $95 \%$ confidence interval of $3 \mathrm{~d}$ food record data by category of food items from the short FFQ among pre-school children ( $n$ 64) aged 2-5 years, metropolitan Sydney, Australia, September 2007-April 2008

\begin{tabular}{|c|c|c|c|c|c|c|c|c|c|}
\hline \multirow{3}{*}{$\begin{array}{l}\text { FFQ food item } \\
\text { Vegetables }\end{array}$} & \multicolumn{8}{|c|}{ Survey response categories } & \multirow[t]{3}{*}{$P$ for trendt } \\
\hline & \multicolumn{2}{|c|}{$\leq 4$ servings/week ( $n$ 13) } & \multicolumn{2}{|c|}{$>4-7$ servings/week $(n 21)$} & \multicolumn{2}{|c|}{$1 \cdot 5-2$ servings/d (n 23) } & \multicolumn{2}{|c|}{$>2$ servings/d (n 7) } & \\
\hline & Mean & $95 \% \mathrm{Cl}$ & Mean & $95 \% \mathrm{Cl}$ & Mean & $95 \% \mathrm{Cl}$ & Mean & $95 \% \mathrm{Cl}$ & \\
\hline Servings/d & $0 \cdot 7$ & $0 \cdot 4,1 \cdot 0$ & 0.9 & $0 \cdot 6,1 \cdot 3$ & $1 \cdot 8$ & $1 \cdot 3,2 \cdot 2$ & $1 \cdot 8$ & $1 \cdot 1,2 \cdot 5$ & $<0.0001$ \\
\hline Fibre $(\mathrm{g})$ & 16 & 12,20 & 17 & 15,19 & 18 & 16,19 & 19 & 16,21 & $0 \cdot 15$ \\
\hline Vitamin C (mg) & 56 & 28,84 & 84 & 46,123 & 111 & 89,132 & 99 & 61,136 & 0.03 \\
\hline Folate $(\mu \mathrm{g})$ & 229 & 166,291 & 206 & 178,234 & 223 & 198,248 & 234 & 198,269 & $0 \cdot 76$ \\
\hline \multirow[t]{2}{*}{ Fruits } & \multicolumn{2}{|c|}{$\leq 1$ serving/d $(n 16)$} & \multicolumn{2}{|c|}{$1 \cdot 5-2$ servings/d ( $n$ 25) } & \multicolumn{2}{|c|}{$2 \cdot 5-3$ servings/d $(n 16)$} & \multicolumn{2}{|c|}{$>3$ servings/d $(n 7)$} & \\
\hline & Mean & $95 \% \mathrm{Cl}$ & Mean & $95 \% \mathrm{Cl}$ & Mean & $95 \% \mathrm{Cl}$ & Mean & $95 \% \mathrm{Cl}$ & \\
\hline Servings/d & $1 \cdot 0$ & $0.6,1 \cdot 4$ & $1 \cdot 3$ & $0 \cdot 9,1 \cdot 7$ & $1 \cdot 8$ & $1 \cdot 2,2 \cdot 3$ & $2 \cdot 8$ & $1 \cdot 9,3 \cdot 7$ & $<0.0001$ \\
\hline Fibre (g) & 16 & 12,19 & 17 & 15,18 & 17 & 15,20 & 22 & 19,24 & 0.01 \\
\hline Vitamin C (mg) & 83 & 29,137 & 87 & 66,109 & 101 & 76,125 & 88 & 59,117 & 0.59 \\
\hline \multirow[t]{2}{*}{ Red meats } & \multicolumn{2}{|c|}{$\leq 1$ time/week (n 16) } & \multicolumn{2}{|c|}{ 1-2 times/week ( $n$ 8) } & \multicolumn{2}{|c|}{$>2-3$ times/week ( $n$ 19) } & \multicolumn{2}{|c|}{$>3$ times/week ( $n$ 21) } & \\
\hline & Mean & $95 \% \mathrm{Cl}$ & Mean & $95 \% \mathrm{Cl}$ & Mean & $95 \% \mathrm{Cl}$ & Mean & $95 \% \mathrm{Cl}$ & \\
\hline Frequency/d & 0.2 & $0 \cdot 1,0 \cdot 4$ & 0.3 & $0.1,0.6$ & 0.4 & $0.3,0.5$ & $0 \cdot 4$ & $0.2,0.6$ & $0 \cdot 17$ \\
\hline Weight $(\mathrm{g})$ & & 4,30 & 31 & 4,58 & 26 & 12,40 & 32 & 11,53 & 0.29 \\
\hline Protein (g) & & 54,67 & 63 & 47,79 & 59 & 54,65 & 70 & 62,79 & 0.12 \\
\hline $\mathrm{Zn}(\mathrm{mg})$ & $7 \cdot 7$ & $7 \cdot 0,8 \cdot 3$ & $8 \cdot 5$ & $6 \cdot 5,10 \cdot 5$ & $7 \cdot 6$ & $7 \cdot 0,8 \cdot 3$ & $9 \cdot 3$ & $8 \cdot 1,10 \cdot 6$ & 0.06 \\
\hline \multirow[t]{2}{*}{ Processed meats } & $\leq 2$ time & lonth (n 16) & 1 tim & ek $(n 17)$ & $2-3$ tim & week (n 16) & $>3$ time & /week (n 15) & \\
\hline & Mean & $95 \% \mathrm{Cl}$ & Mean & $95 \% \mathrm{Cl}$ & Mean & $95 \% \mathrm{Cl}$ & Mean & $95 \% \mathrm{Cl}$ & \\
\hline Frequency/d & 0.4 & $0.2,0.6$ & 0.4 & $0.2,0.6$ & 0.5 & $0.2,0.7$ & 0.5 & $0 \cdot 3,0.7$ & 0.34 \\
\hline Weight $(\mathrm{g})$ & 14 & 7,22 & 21 & 10,31 & 19 & 5,33 & 21 & 9,34 & 0.43 \\
\hline Energy (kJ) & 5976 & 5379,6573 & 5727 & 5219,6573 & 7014 & 6187,7841 & 6686 & 5888,7485 & 0.02 \\
\hline Protein (g) & 61 & 52,71 & 57 & 50,63 & 70 & 60,78 & 69 & 61,77 & 0.05 \\
\hline Total fat $(\mathrm{g})$ & 52 & 43,60 & 51 & 47,60 & 58 & 50,65 & 58 & 50,65 & $0 \cdot 12$ \\
\hline $\mathrm{Zn}(\mathrm{mg})$ & $8 \cdot 0$ & $6 \cdot 7,9 \cdot 4$ & $7 \cdot 5$ & $6 \cdot 9,8 \cdot 2$ & $9 \cdot 0$ & $8 \cdot 0,10 \cdot 1$ & $8 \cdot 6$ & $7 \cdot 3,9 \cdot 9$ & $0 \cdot 16$ \\
\hline High-fat potatoes & $<1 \mathrm{tim}$ & onth (n 15) & 1 time & nth $(n$ 15) & $2-3$ time & nonth (n 12) & $>1$ time & week (n 22) & \\
\hline & Mean & $95 \% \mathrm{Cl}$ & Mean & $95 \% \mathrm{Cl}$ & Mean & $95 \% \mathrm{Cl}$ & Mean & $95 \% \mathrm{Cl}$ & \\
\hline Frequency/d & 0.04 & $-0.02,0.11$ & 0.04 & $-0.02,0.11$ & 0.03 & $-0.03,0.09$ & 0.12 & $0.01,0.23$ & $0 \cdot 17$ \\
\hline Weight $(\mathrm{g})$ & $2 \cdot 2$ & $-1 \cdot 1,5 \cdot 5$ & $5 \cdot 9$ & $-3 \cdot 2,15 \cdot 1$ & $1 \cdot 2$ & $-1 \cdot 5,3.9$ & $9 \cdot 2$ & $-0.9,19 \cdot 3$ & 0.27 \\
\hline Energy (kJ) & 5964 & 5513,6416 & 6719 & 5920,7518 & 6692 & 5915,7468 & 6134 & 5412,6856 & 0.94 \\
\hline Total fat $(\mathrm{g})$ & 50 & 44,57 & 57 & 50,64 & 60 & 51,68 & 53 & 46,59 & 0.62 \\
\hline Salty snacks & $<1 \mathrm{tim}$ & onth (n 19) & 1 time & nth (n 13) & $2-3$ time & month (n 10) & $>1$ time & week (n 22) & \\
\hline & Mean & $95 \% \mathrm{Cl}$ & Mean & $95 \% \mathrm{Cl}$ & Mean & $95 \% \mathrm{Cl}$ & Mean & $95 \% \mathrm{Cl}$ & \\
\hline Frequency/d & 0.09 & $0.00,0.18$ & 0.03 & $-0.03,0.08$ & 0.07 & $-0.03,0.17$ & 0.11 & $0.04,0.18$ & 0.55 \\
\hline Weight $(\mathrm{g})$ & $2 \cdot 2$ & $-0.1,4 \cdot 6$ & 0.5 & $-0.6,1.6$ & 0.8 & $-0.7,2 \cdot 3$ & $4 \cdot 7$ & $0 \cdot 1,9 \cdot 3$ & $0 \cdot 21$ \\
\hline Energy (kJ) & 6399 & 5778,7021 & 6087 & 5402, 6772 & 6724 & 5399,8049 & 6252 & 5652,6853 & 0.94 \\
\hline Total fat $(\mathrm{g})$ & 56 & 49,63 & 51 & 42,60 & 55 & 45,65 & 55 & 50,60 & 0.97 \\
\hline Biscuits & $<1$ tim & eek ( $n$ 11) & $1-2$ tim & veek (n 15) & 3-6 tim & week (n 13) & $\geq 1 \mathrm{tin}$ & e/d (n 25) & \\
\hline & Mean & $95 \% \mathrm{Cl}$ & Mean & $95 \% \mathrm{Cl}$ & Mean & $95 \% \mathrm{Cl}$ & Mean & $95 \% \mathrm{Cl}$ & \\
\hline Frequency/d & 0.9 & $0 \cdot 5,1 \cdot 2$ & $1 \cdot 8$ & $1 \cdot 2,2 \cdot 3$ & $1 \cdot 3$ & $0 \cdot 9,1 \cdot 7$ & $1 \cdot 5$ & $1 \cdot 2,1 \cdot 9$ & $0 \cdot 15$ \\
\hline Weight $(\mathrm{g})$ & 18 & 8,28 & 42 & 26,58 & 44 & 22,65 & 37 & 26,48 & 0.17 \\
\hline Energy (kJ) & 6217 & 5101, 7332 & 5835 & 5320,6345 & 6682 & 5603,7761 & 6509 & 6024, 6994 & 0.24 \\
\hline Total fat $(\mathrm{g})$ & 56 & 43,68 & 49 & 43,55 & 58 & 48,68 & 55 & 51,59 & 0.60 \\
\hline Saturated fat $(\mathrm{g})$ & 24 & 17,31 & 22 & 19,25 & 27 & 22,33 & 26 & 24,28 & $0 \cdot 19$ \\
\hline Sugars (g) & 79 & 62,96 & 81 & 72,91 & 96 & 77,115 & 100 & 88,111 & 0.01 \\
\hline Confectionery & $<1$ tim & leek ( $n$ 18) & $1-1.5 \mathrm{tir}$ & week (n 17) & $2-3$ tim & week (n 14) & $\geq 4$ time & /week (n 15) & \\
\hline & Mean & $95 \% \mathrm{Cl}$ & Mean & $95 \% \mathrm{Cl}$ & Mean & $95 \% \mathrm{Cl}$ & Mean & $95 \% \mathrm{Cl}$ & \\
\hline Frequency/d & $0 \cdot 3$ & $0.0,0.5$ & 0.3 & $0.2,0.5$ & 0.6 & $0 \cdot 2,1 \cdot 1$ & 0.5 & $0.3,0.7$ & 0.09 \\
\hline Weight $(\mathrm{g})$ & $2 \cdot 7$ & $-0 \cdot 1,5 \cdot 6$ & $3 \cdot 6$ & $1 \cdot 4,5 \cdot 9$ & $6 \cdot 1$ & $2 \cdot 1,10 \cdot 2$ & $6 \cdot 6$ & $3 \cdot 0,10 \cdot 2$ & 0.04 \\
\hline Energy (kJ) & 6168 & 5553,6783 & 6747 & 6056,7437 & 6796 & 5802,7790 & 5643 & 5168,6117 & 0.34 \\
\hline Sugars (g) & 89 & 73,104 & 89 & 72,105 & 104 & 90,118 & 84 & 75,93 & 0.97 \\
\hline
\end{tabular}

tOne-way ANOVA with test for linearity. 
Table 3 Relative validity: mean and 95\% confidence interval of $3 \mathrm{~d}$ food record by category of beverages from the short FFQ among preschool children ( $n$ 64) aged 2-5 years, metropolitan Sydney, Australia, September 2007-April 2008

\begin{tabular}{|c|c|c|c|c|c|c|c|c|c|}
\hline \multirow{3}{*}{$\begin{array}{l}\text { FFQ beverage item } \\
\text { Milk }\end{array}$} & \multicolumn{8}{|c|}{ Survey response categories } & \multirow[t]{3}{*}{$P$ for trendt } \\
\hline & \multicolumn{2}{|c|}{$<1 \mathrm{cup} / \mathrm{d}(n 10)$} & \multicolumn{2}{|c|}{$1-1.5$ cups/d (n 19) } & \multicolumn{2}{|c|}{2 cups/d ( $n$ 19) } & \multicolumn{2}{|c|}{$\geq 2.5 \mathrm{cups} / \mathrm{d}(n 16)$} & \\
\hline & Mean & $95 \% \mathrm{Cl}$ & Mean & $95 \% \mathrm{Cl}$ & Mean & $95 \% \mathrm{Cl}$ & Mean & $95 \% \mathrm{Cl}$ & \\
\hline Cups/d & 0.8 & $0 \cdot 3,1 \cdot 3$ & $1 \cdot 6$ & $1 \cdot 3,2 \cdot 0$ & $1 \cdot 5$ & $1 \cdot 3,1 \cdot 7$ & $2 \cdot 1$ & $1 \cdot 6,2 \cdot 7$ & $<0.001$ \\
\hline Protein (g) & 62 & 53,71 & 63 & 55,70 & 62 & 58,67 & 67 & 54,80 & 0.41 \\
\hline Total fat $(\mathrm{g})$ & 48 & 39,57 & 55 & 49,62 & 52 & 46,58 & 60 & 52,68 & 0.07 \\
\hline Saturated fat $(\mathrm{g})$ & 21 & 17,25 & 25 & 22,28 & 24 & 20,27 & 29 & 24,33 & 0.01 \\
\hline $\mathrm{Ca}(\mathrm{mg})$ & 714 & 461,967 & 1006 & 850,1162 & 893 & 799,987 & 1085 & 900,1270 & 0.02 \\
\hline$P(m g)$ & 1103 & 869,1337 & 1282 & 1125,1439 & 1159 & 1072,1246 & 1391 & 1151,1631 & $0 \cdot 10$ \\
\hline \multirow[t]{2}{*}{ Soft drinksł } & \multicolumn{2}{|c|}{ Does not drink ( $n$ 39) } & \multicolumn{2}{|c|}{$<1$ cup/week $(n 9)$} & \multicolumn{2}{|c|}{$1-3$ cups/week ( $n$ 10) } & \multicolumn{2}{|c|}{$\geq 3.5$ cups/week $(n 6)$} & \\
\hline & Mean & $95 \% \mathrm{Cl}$ & Mean & $95 \% \mathrm{Cl}$ & Mean & $95 \% \mathrm{Cl}$ & Mean & $95 \% \mathrm{Cl}$ & \\
\hline Cups/d & $0 \cdot 1$ & $0 \cdot 0,0 \cdot 1$ & $0 \cdot 1$ & $-0 \cdot 1,0 \cdot 3$ & 0.0 & $0 \cdot 0,0 \cdot 1$ & $0 \cdot 4$ & $0 \cdot 0,0 \cdot 8$ & 0.01 \\
\hline Energy (kJ) & 6252 & 5873,6631 & 6599 & 5391,7807 & 6125 & 5333,6917 & 6838 & 4194, 9481 & 0.53 \\
\hline Sugars $(\mathrm{g})$ & 89 & 82,96 & 97 & 68,126 & 89 & 63,115 & 97 & 60,134 & 0.59 \\
\hline \multirow[t]{2}{*}{ Diet soft drinks } & \multicolumn{2}{|c|}{ Does not drink ( $n$ 61) } & \multicolumn{2}{|c|}{$<1$ cup/week $(n 0)$} & \multicolumn{2}{|c|}{$1-3$ cups/week $(n 2)$} & \multicolumn{2}{|c|}{$\geq 3.5$ cups/week $(n 1)$} & \\
\hline & Mean & $95 \% \mathrm{Cl}$ & Mean & $95 \% \mathrm{Cl}$ & Mean & $95 \% \mathrm{Cl}$ & Mean & $95 \% \mathrm{Cl}$ & \\
\hline Cups/d & $0 \cdot 0$ & $0.0,0.02$ & & IA & $0 \cdot 25$ & $0,1 \cdot 0$ & & JA & $0 \cdot 0001$ \\
\hline \multirow[t]{2}{*}{ Fruit juices } & \multicolumn{2}{|c|}{$\leq 1$ cup/month ( $n$ 18) } & \multicolumn{2}{|c|}{$0.5-3$ cups/week ( $n$ 23) } & \multicolumn{2}{|c|}{$0.5-1 \mathrm{cup} / \mathrm{d}(n 15)$} & \multicolumn{2}{|c|}{$\geq 1.5 \mathrm{cups} / \mathrm{d}(n 8)$} & \\
\hline & Mean & $95 \% \mathrm{Cl}$ & Mean & $95 \% \mathrm{Cl}$ & Mean & $95 \% \mathrm{Cl}$ & Mean & $95 \% \mathrm{Cl}$ & \\
\hline Cups/d & $0 \cdot 1$ & $0 \cdot 0,0 \cdot 2$ & $0 \cdot 1$ & $0 \cdot 0,0 \cdot 3$ & 0.4 & $0.3,0.6$ & $0 \cdot 7$ & $0 \cdot 2,1 \cdot 1$ & $<0.001$ \\
\hline Sugars (g) & 87 & 76,98 & 86 & 74,98 & 90 & 78,102 & 117 & 85,1496 & 0.03 \\
\hline Vitamin C (mg) & 65 & 40,90 & 78 & 58,98 & 112 & 87,138 & 135 & 33,237 & 0.003 \\
\hline $\mathrm{K}(\mathrm{mg})$ & 2316 & 1952, 2680 & 2305 & 2093,2518 & 2372 & 2090,2654 & 2802 & 2054,3549 & $0 \cdot 13$ \\
\hline \multirow[t]{2}{*}{ Water } & \multicolumn{2}{|c|}{$\leq 1.5 \mathrm{cups} / \mathrm{d}(n+15)$} & \multicolumn{2}{|c|}{$2-2 \cdot 5$ cups/d (n 15) } & \multicolumn{2}{|c|}{$3-3.5$ cups/d ( $n$ 16) } & \multicolumn{2}{|c|}{$\geq 4$ cups/d ( $n$ 18) } & \\
\hline & Mean & $95 \% \mathrm{Cl}$ & Mean & $95 \% \mathrm{Cl}$ & Mean & $95 \% \mathrm{Cl}$ & Mean & $95 \% \mathrm{Cl}$ & \\
\hline Cups/d & $1 \cdot 2$ & $0 \cdot 7,1 \cdot 7$ & $1 \cdot 5$ & $0 \cdot 8,2 \cdot 2$ & $1 \cdot 7$ & $1 \cdot 0,2 \cdot 3$ & $2 \cdot 3$ & $1 \cdot 6,2 \cdot 9$ & 0.01 \\
\hline
\end{tabular}

NA, not applicable.

tOne-way ANOVA with test for linearity.

‡lncludes soft drinks, syrups and sports drinks.

water) had significant $P$ values for trend. The beverage of milk had similar absolute quantities reported in the FFQ and the FR: among people reporting consumption of 2.5 cups or more of milk daily in the FFQ, the mean number of cups of milk from their FR was $2 \cdot 1 \mathrm{cups} / \mathrm{d}$, compared with $0.8 \mathrm{cups} / \mathrm{d}$ among those who reported consuming less than one cup daily $(P<0 \cdot 001)$. However, the other drinks had significant $P$ values for trend, but they did not equate well in absolute quantities: people who reported consuming four or more cups of water daily in the FFQ consumed $2 \cdot 3 \mathrm{cups} / \mathrm{d}$ in the FR, compared with $1 \cdot 2$ cups/d among those who reported consuming less than 1.5 cups of water daily. Among those reporting increasing cups of milk, there was a significant $P$ value for trend for $\mathrm{Ca}(P$ for trend $=0 \cdot 02)$ and saturated fat intake $(0 \cdot 01)$; among those reporting increasing cups of juice, there was a significant $P$ value for trend for total sugars $(0 \cdot 03)$ and vitamin $C(0 \cdot 003)$.

The mean and median amounts of food and beverage items reported in the FFQ and consumed in the FR are presented in Table 4. Fruit, fruit juice and water were significantly higher in the FFQ than the FR $(P<0 \cdot 001)$, and processed meats and biscuits were significantly lower in the FFQ than the FR $(P<0 \cdot 01)$. There was no significant difference between the FFQ and FR for vegetables, red meat, high-fat potatoes, confectionery, soft drinks (sugary and diet) and milk. Rank correlation coefficients were $>0.5$ for vegetables, fruit, diet soft drinks and fruit juice.

The sensitivity to identify two or more servings of vegetables from the FFQ was $83 \%$ (95\% CI 52, 98\%) and the specificity was $63 \%$ (95\% CI 49, 76\%), with positive predictive value of $34 \%$ (95\% CI 18, 54\%) and negative predictive value of $94 \%$ (95\% CI 81, 99\%). The sensitivity to identify one or more servings of fruit from the FFQ was 98\% (95\% CI 88, 100\%), specificity was 17\% (95\% CI 4, $41 \%$ ), positive predictive value was $75 \%$ (95\% CI 62, $85 \%$ ) and negative predictive value was $75 \%$ (95\% CI 19, 99\%).

Bland-Altman plots (Fig. 1) were constructed to describe the agreement between the two methods for servings of fruit and vegetables and cups of water and milk. All plots indicated a positive mean difference between the short FFQ and FR, since for these food items 
Table 4 Comparison of mean and median intakes, mean difference and rank correlation between the short FFQ and $3 \mathrm{~d}$ food record (FR) among pre-school children ( $n$ 64) aged 2-5 years, metropolitan Sydney, Australia, September 2007-April 2008

\begin{tabular}{|c|c|c|c|c|c|c|c|}
\hline \multirow[b]{2}{*}{ FFQ item } & \multicolumn{2}{|c|}{$\mathrm{FFQ}$} & \multicolumn{2}{|c|}{ FR } & \multirow[b]{2}{*}{ Mean difference } & \multirow[b]{2}{*}{ SED } & \multirow{2}{*}{$\begin{array}{c}\text { Spearman's rank } \\
\text { correlation }\end{array}$} \\
\hline & Mean & Median & Mean & Median & & & \\
\hline Vegetables (servings/d) & $1 \cdot 4$ & $1 \cdot 0$ & $1 \cdot 3$ & $1 \cdot 2$ & $0 \cdot 12$ & $0 \cdot 11$ & $0 \cdot 55^{\star \star \star}$ \\
\hline Fruits (servings/d) & $2 \cdot 1$ & $2 \cdot 0$ & $1 \cdot 5$ & $1 \cdot \overline{3}$ & $0.64^{\star \star *}$ & $0 \cdot 13$ & $0.52^{\star \star \star}$ \\
\hline Red meats (frequency/d) & $0 \cdot 4$ & $0 \cdot 4$ & $0 \cdot 3$ & $0 \cdot 3$ & $0 \cdot 10$ & 0.06 & $0 \cdot 16$ \\
\hline Processed meats (frequency/d) & $0 \cdot 26$ & $0 \cdot 14$ & 0.43 & 0.33 & $-0 \cdot 17^{\star \star}$ & 0.05 & $0 \cdot 14$ \\
\hline High-fat potatoes (frequency/d) & 0.08 & 0.06 & 0.07 & $0 \cdot 0$ & 0.01 & 0.02 & $0 \cdot 17$ \\
\hline Salty snacks (frequency/d) & $0 \cdot 12$ & 0.05 & 0.08 & $0 \cdot 0$ & 0.04 & 0.03 & $0 \cdot 15$ \\
\hline Biscuits (frequency/d) & 0.6 & 0.5 & $1 \cdot 4$ & $1 \cdot 3$ & $-0 \cdot 80^{\star \star *}$ & $0 \cdot 11$ & $0 \cdot 26^{*}$ \\
\hline Confectionery (frequency/d) & 0.33 & $0 \cdot 14$ & 0.33 & 0.14 & -0.09 & 0.07 & $0 \cdot 38^{\star *}$ \\
\hline Milk (cups/d) & $1 \cdot 8$ & $2 \cdot 0$ & $1 \cdot 6$ & $1 \cdot 4$ & $0 \cdot 19$ & $0 \cdot 13$ & $0 \cdot 41^{\star *}$ \\
\hline Soft drinks (cups/d) & $0 \cdot 13$ & 0 & 0.05 & 0 & 0.05 & 0.04 & $0 \cdot 29^{*}$ \\
\hline Diet soft drinks (cups/d) & $0 \cdot 05$ & 0 & 0.03 & 0 & $0 \cdot 24$ & 0.03 & $0.67^{\star \star \star}$ \\
\hline Fruit juices (cups/d) & $0 \cdot 56$ & 0.25 & 0.26 & $0 \cdot 15$ & $0 \cdot 30^{\star \star \star}$ & 0.08 & $0.59^{\star \star \star}$ \\
\hline Water (cups/d) & $2 \cdot 8$ & $3 \cdot 0$ & $1 \cdot 7$ & $1 \cdot 2$ & $1 \cdot 15^{\star \star \star}$ & 0.20 & $0 \cdot 35^{\star *}$ \\
\hline
\end{tabular}

${ }^{\star} P<0.05,{ }^{* \star} P<0.01,{ }^{* \star *} P<0.001$ based on paired $t$ tests.
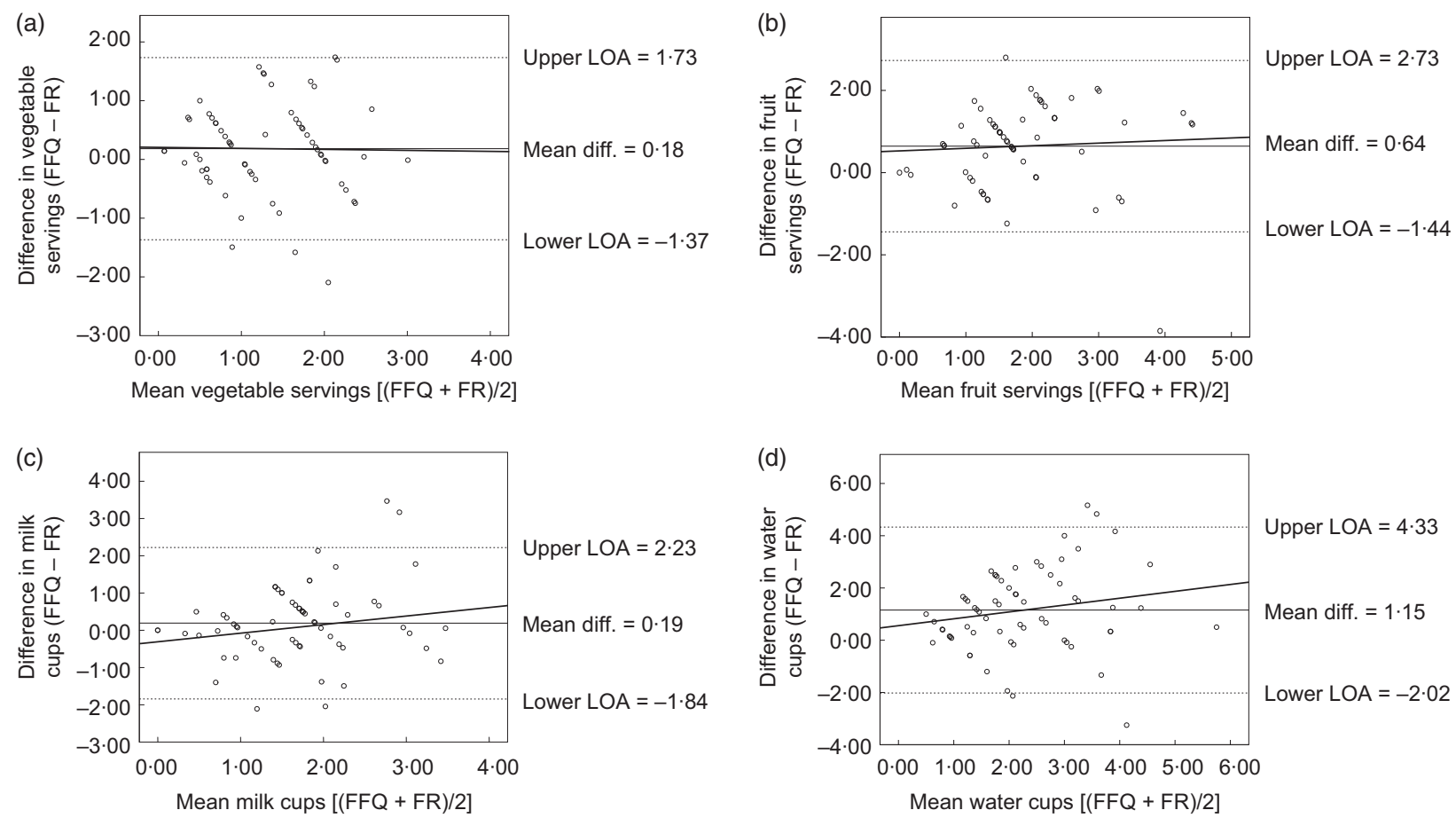

Fig. 1 Bland-Altman plots assessing the validity of the first FFQ $v$. the $3 \mathrm{~d}$ food record (FR) for intakes of vegetables, fruit, milk and water among pre-school children ( $n$ 64) aged 2-5 years, metropolitan Sydney, Australia, September 2007-April 2008. Plots show the mean difference (Mean diff.; - ), the $95 \%$ limits of agreement (LOA; --- ) and the fitted regression lines $(-)$ for: (a) servings of vegetables (difference vegetable servings $=0.21+-0.02 \times$ mean vegetable servings $(95 \% \mathrm{Cl}-0.30,0.27), P$ for linear trend $=0.91$ ); (b) servings of fruit (difference fruit servings $=0.53+0.06 \times$ mean fruit servings $(95 \% \mathrm{Cl}-0.21,0.34), P$ for linear trend $=0.65)$; (c) cups of milk (difference milk cups $=-0.31+0.30 \times$ mean milk cups $(95 \% \mathrm{Cl}-0.21,0.62)$, $P$ for linear trend $=0.07$ ); and (d) cups of water (difference water cups $=0.56+0.26 \times$ mean water cups $(95 \% \mathrm{Cl}-0.09,0 \cdot 62), P$ for linear trend $=0 \cdot 15)$

the short FFQ tended to provide a higher estimate of foods consumed than the FR. Most measurements fell within the $95 \%$ limits of agreement as expected, and there were no significant linear trends for the fitted regression lines, indicating no systematic bias between the two methods. Because the responses for servings of fruit and vegetables and cups of milk and water from the seventeen-item FFQ tend to be integers, whereas the FR has a range of continuous data, the Bland-Altman plots tend to fall in diagonal lines.

\section{Discussion}

The present study investigated the repeatability and validity of a short FFQ proxy reported by parents of children of 
pre-school age to assess selected food consumption and behaviours in this age group. A range of food consumption habits had moderate to good repeatability and selected questions had good validity, especially fruit and vegetable servings and drinks. Water and sugary drinks did not perform well in terms of absolute quantity of intake; however, they were able to provide information about the ranking of foods and nutrients.

Although other questions did not perform well for direct validity, they were still able to provide significant indirect validity for selected nutrients, such as occurred with red meat (protein, Zn), processed meat (energy, protein and $\mathrm{Zn}$ ) and snack foods (sugars). Likewise, in validity analyses undertaken of short questions as part of the Australian National Food and Nutrition Monitoring and Surveillance Project, among a group of 766 adults, those who reported more frequent consumption of red meat and processed meat consumed more meat in $3 \mathrm{~d}$ weighed food records and also had increasingly higher intakes of energy, protein, $\mathrm{Zn}$, fat and saturated fat $(P<0 \cdot 05)^{(24)}$.

Information about the sensitivity and specificity of fruit and vegetable questions from the FFQ provides useful information to consider when FFQ data are used to report the proportion of people meeting recommended servings. The high sensitivity for both fruit and vegetables ( $83 \%$ and $98 \%$ respectively) indicates that the FFQ will detect most of those who meet the guidelines according to the FR. However, because the FFQ overestimates consumption of both, its specificity is quite low for vegetables (63\%) and poor for fruit (17\%) which we showed was very significantly overestimated by the FFQ (Table 4). As a result the FFQ will fail to identify many children who are not meeting the fruit guidelines (83\%). From the positive predictive values we see that if the FFQ indicates a child is meeting the vegetable guidelines there is a relatively low probability that he/she really is (34\%), while this probability is higher for fruit (75\%). On the other hand, the negative predictive values show that if the FFQ indicates a child is not meeting the vegetable guidelines then he/she very likely really is not (94\%), while the very wide confidence intervals for the negative predictive value for fruit $(75 \%)$ reflect the fact that the FFQ identified only four children who did not meet the fruit guidelines and three of them really did not.

The Bland-Altman plots for fruit, vegetables, water and milk indicated no bias as the mean intake increases or decreases. This shows that the FFQ is most useful when measuring and comparing dietary intakes of children at a population level, rather than at an individual level.

The current study of pre-school children provides useful information to support population health monitoring and, importantly, may be used in the assessment of diet in larger research programmes. Keeping detailed food records or undertaking $24 \mathrm{~h}$ recalls of dietary consumption can be burdensome for participants and resource-intensive for researchers, so it is appealing to have a simpler and shorter tool. Our findings have some similarities to a study of 4-16-year-old children in which a twenty-eight-item questionnaire, known as the Children's Dietary Questionnaire (CDQ), was tested for repeatability and relative validity $^{(8)}$. In that study $(n 709)$, the repeatability values for fruit and vegetable intake and non-core foods were reasonable, but fat from dairy and sweetened beverages had poorer repeatability. The authors concluded that the relative validity of the CDQ, assessed using a $7 \mathrm{~d}$ frequency checklist completed by the carers and parents of the children, was suitable only to assess habits at a group level and was not suitable to measure change in individual intakes ${ }^{(8)}$. In the case of the CDQ, the authors used a short frequency tool as a comparison dietary assessment method, rather than the more common method of food record or multiple $24 \mathrm{~h}$ recalls $^{(28)}$. The CDQ was also used to score the diet habits, unlike the tool under investigation in the current research.

In the European study of 2-9-year-old children ( $n$ 258) of the Children's Eating Habits Questionnaire (CEHQ-FFQ), forty-three food items were included in the FFQ and the reproducibility of the FFQ was assessed, with reproducibility lowest for diet soft drinks $(\kappa=0 \cdot 23, r=0 \cdot 32)$ and highest for sweetened milk $(\kappa=0 \cdot 68, r=0 \cdot 76)^{(7)}$. In our study, the food item with the poorest repeatability was red meat $\left(\kappa_{\mathrm{w}}=0 \cdot 37, \mathrm{ICC}=0 \cdot 27\right)$, with the best measure being the reported frequency of take-away foods $\left(\kappa_{\mathrm{w}}=0.85\right.$, ICC $=0 \cdot 77$ ). No assessment of validity was performed by the European study of 2-9-year-old children.

In the Belgian study of pre-school children ${ }^{(6)}$, food items with Spearman rank correlation coefficient $>0.5$ between the FFQ and the diet record were fruit, milk products, cheese, sugared drinks and fruit juice, with similar in our own study for fruit and fruit juice but not for the other items. In the Canadian study of pre-school children, the FFQ was used to provide an overall score of diet quality to determine the nutritional risk in pre-schoolers, and this was validated and compared with results from $3 \mathrm{~d}$ dietary records/recalls ${ }^{(5)}$. That study is not directly comparable to our study, in which we investigated the validity and repeatability of individual food items, rather than an overall score of diet quality.

There are some limitations to the present study. First, a number of the short diet questions did not perform well in terms of their relationship to a more detailed dietary assessment method, in particular questions about red meat, processed meat, hot chips, salty snacks (e.g. potato chips) and snack foods (includes biscuits, cakes, doughnuts, muesli bars). This could be due to a number of factors. The parents who participated in our study were mostly tertiary educated and generally the children recorded healthy diets, with a limited intake of energy-dense, nutrient-poor foods. It is likely that those who participated in the study were well motivated parents, who carefully provided mostly healthy foods for their children. The relatively small weights recorded of energy-dense nutrient-poor foods made it difficult to assess the validity of the categorical data 
from the questions. For example, in the salty snacks question, those who reported consuming salty snacks more than once weekly $(22 / 64,34 \%)$ had a daily average intake of $4 \cdot 2 \mathrm{~g}$ of salty snacks compared with $2 \cdot 2 \mathrm{~g}$ of salty snacks among those who reported consuming less than once monthly. Both mean values were relatively low, limiting the interpretability of the findings. Additionally, some of the short questions required parents and carers to consider multiple factors at once, which may be difficult to calculate accurately and quickly. An example is the snack food question, where respondents are asked to report frequency of consumption of biscuits, cakes, doughnuts or muesli bars within the same question. It would probably be easier to answer this question if it included fewer different foods within the one question.

A second limitation is the relatively small total number of participants, which means for each category of response that there were fairly small numbers to compare, limiting the generalisability of the findings.

Third, given the FR was limited to $3 \mathrm{~d}$, there may be less ability to capture accurate information about less frequently consumed food items, such as high-fat potatoes and salty snacks, and indeed food items which were consumed daily (e.g. fruit, vegetables, and beverages) produced a higher correlation between the FFQ and FR. It may be useful to consider recording more days in future analyses of validity in order to better capture habitual intake; however, this needs to be balanced against possible problems with increasing fatigue and boredom associated with recording ${ }^{(22)}$.

\section{Conclusion}

The present study of the repeatability and validity of a short FFQ among children aged 2-5 years, reported by their parents and pre-school teachers, indicated good to moderate repeatability for most questions and moderate validity for short questions about fruit and vegetable servings and different types of drinks consumed. Other questions about dietary habits, which asked about frequency of consumption, had poorer validity. This information is useful for interpreting short questions used in monitoring of food intake in populations, and also could be used in population-based research to assess response to interventions ${ }^{(29)}$. It should be noted that the participating parents were mostly tertiary educated, with English as their first language, so these findings may not be representative of other subgroups in the population.

\section{Acknowledgements}

Sources of funding: The current research is related to work of the Healthy Beginnings Trial (HBT) funded by the Australian National Health and Medical Research Council (ID number: 393112); however the participants of the present study were not part of the HBT. Conflicts of interest: There are no conflicts of interest. Authors' contribution: V.M.F. conceived the idea of the study, undertook the literature review, data analysis and interpretation, and wrote the original draft. V.M.F. and L.L.H. contributed to the study design and acquisition of data; J.M.S. provided advice on data analysis; L.M.W., L.L.H., J.M.S., C.R. and L.A.B. made significant comments on the draft. All authors have read and approved the final manuscript. Acknowledgements: The authors thank the research assistants who assisted with dietary data collection and data entry: Ms Kristy McDonald and Dr Jimmy Louie. They also thank Mr Aaron Thrift and Professor Jennifer Peat who provided advice about statistical analyses.

\section{References}

1. Willett WC, Sampson L, Stampfer MJ et al. (1985) Reproducibility and validity of a semiquantitative food frequency questionnaire. Am J Epidemiol 122, 51-65.

2. Block G, Hartman AM, Dresser CM et al. (1986) A data-based approach to diet questionnaire design and testing. Am J Epidemiol 124, 453-469.

3. Watson JF, Collins CE, Sibbritt DW et al. (2009) Reproducibility and comparative validity of a food frequency questionnaire for Australian children and adolescents. Int J Behav Nutr Phys Act 6, 62.

4. Gwynn JD, Flood VM, D'Este CA et al. (2011) The reliability and validity of a short FFQ among Australian Aboriginal and Torres Strait Islander and non-Indigenous rural children. Public Health Nutr 14, 388-401.

5. Simpson JAR, Keller HH, Rysdale LA et al. (2008) Nutrition Screening Tool for Every Preschooler (NutriSTEP ${ }^{\mathrm{TM}}$ ): validation and test-retest reliability of a parent-administered questionnaire assessing nutrition risk of preschoolers. Eur J Clin Nutr 62, 770-780.

6. Huybrechts I, De Backer G, De Bacquer D et al. (2009) Relative validity and reproducibility of a food-frequency questionnaire for estimating food intakes among Flemish preschoolers. Int J Environ Res Public Health 6, 382-399.

7. Lanfer A, Hebestreit A, Ahrens W et al. (2011) Reproducibility of food consumption frequencies derived from the Children's Eating Habits Questionnaire used in the IDEFICS study. Int J Obes (Lond) 35, Suppl. 1, S61-S68.

8. Magarey A, Golley R, Spurrier N et al. (2009) Reliability and validity of the Children's Dietary Questionnaire; a new tool to measure children's dietary patterns. Int J Pediatr Obes $\mathbf{4}$, 257-265.

9. Baranowski T, Smith M, Baranowski J et al. (1997) Low validity of a seven-item fruit and vegetable food frequency questionnaire among third-grade students. J Am Diet Assoc 97, 66-68.

10. Prochaska JJ \& Sallis JF (2004) Reliability and validity of a fruit and vegetable screening measure for adolescents. $J$ Adolesc Health 34, 163-165.

11. Rockett HRH, Berkey CS \& Colditz GA (2007) Comparison of a short food frequency questionnaire with the Youth/ Adolescent Questionnaire in the Growing Up Today Study. Int J Pediatr Obes 2, 31-39.

12. Vereecken CA, Rossi S, Giacchi MV et al. (2008) Comparison of a short food-frequency questionnaire and derived indices with a seven-day diet record in Belgian and Italian children. Int J Public Health 53, 297-305.

13. Lillegaard ITL, Øverby NC \& Andersen LF (2012) Evaluation of a short food frequency questionnaire used among Norwegian children. Food Nutr Res 56 10.3402/fnr.v56i0.6399. 
14. Baranowski T \& Domel SB (1994) A cognitive model of childrens reporting of food intake. Am J Clin Nutr 59, 1 Suppl., S212-S217.

15. Vereecken C, Covents M \& Maes L (2010) Comparison of a food frequency questionnaire with an online dietary assessment tool for assessing preschool children's dietary intake. J Hum Nutr Diet 23, 502-510.

16. Quaine J, Eyeson-Annan M, Baker D et al. (2003) Report on the development of the NSW Child Health Survey 2001. N S W Public Health Bull 14, Suppl. 1.

17. Flood VM, Webb K \& Rangan A (editors) (2005) Recommendations for Short Questions to Assess Food Consumption in Children for the NSW Health Surveys. Sydney: NSW Centre for Public Health Nutrition.

18. Centre for Epidemiology and Research (2009) 2008 Summary Report on Adult Health from the New Wouth Wales Population Health Survey. Sydney: NSW Department of Health.

19. Landis JR \& Koch GG (1977) Measurement of observer agreement for categorical data. Biometrics 33, 159-174.

20. Armitage P, Berry G \& Matthews JNS (2008) Statistical Methods in Medical Research, 4th ed. Oxford: Blackwell.

21. Food Standards Australia New Zealand (1999) AUSNUT (1999). Australian Food and Nutrient Database 1999 for estimation of dietary intake. Canberra: FSANZ.
22. Magarey A, McKean S \& Daniels L (2006) Evaluation of fruit and vegetable intakes of Australian adults: the National Nutrition Survey 1995. Aust N Z J Public Health 30, 32-37.

23. Gibson RS (2005) Principles of Nutritional Assessment, 2nd ed. New York: Oxford University Press.

24. Riley M, Rutishauser I \& Webb K (2001) Comparison of Short Questions with Weighed Dietary Records. Canberra: Australian Food and Nutrition Monitoring Unit.

25. Black AE (2000) Critical evaluation of energy intake using the Goldberg cut-off for energy intake:basal metabolic rate. A practical guide to its calculation, use and limitations. Int J Obes Relat Metab Disord 24, 1119-1130.

26. Morley B, Scully M, Niven P et al. (2010) National Secondary Students' Diet and Activity Survey, 2009-2010: Main Report. Melbourne: Cancer Council Victoria.

27. Bland JM \& Altman DG (1986) Statistical methods for assessing agreement between 2 methods of clinical measurement. Lancet 1, 307-310.

28. Cade JE, Burley VJ, Warm DL et al. (2004) Food-frequency questionnaires: a review of their design, validation and utilisation. Nutr Res Rev 17, 5-22.

29. Wen LM, Baur LA, Simpson JM et al. (2012) Effectiveness of home based early intervention on children's BMI at age 2: randomised controlled trial. $B M J \mathbf{3 4 4}$, e3732. 


\section{Appendix}

Young Children's Food and Drink Study

Thank you for agreeing to participate in this study about short nutrition questions of young children. The following set of short questions will take approximately 10 to 15 min to complete. The answers are confidential and will only be seen by the survey team. No one else will have access to your information. We are interested to learn more about your pre-school child's usual eating habits. I'm going to read you a list of different food and drinks. Please tell me how much of these foods and drinks [child] usually consumes per day or per week.

\section{The first two questions are about fruit and vegetables:}

Q1. How many servings of vegetables does [child's name] usually eat each day? (one serving $=1 / 2$ cup cooked vegetables or 1 cup of salad vegetables)

$\begin{array}{lll}1 . & \text { s. } 1 \text { servings per day } & \text { servings per week }\end{array}$

Q2. How many servings of fruit does [child's name] usually eat each day? (one serving = one medium piece or two small pieces of fruit or one cup of diced pieces) servings per day
2. servings per week
3. Doesn't eat fruit

4. Don't know

5. Refused

\section{The next few questions ask how often your child eats some foods:}

Q3. How often does [child's name] eat red meat, such as beef or lamb? Include all steaks, chops, roasts, mince, stir fries and casseroles. Do not include pork or chicken Longer list (do not read out): Veal, offal (liver, kidney), mutton, game (buffalo, crocodile, goanna, goat, hare, kangaroo, rabbit, snake, venison, wild boar)
times per day
times per week
3. times per month
5.7 don't know/can't say

4. Rarely/never

6. $\overline{\text { Refused }}$

Q4. How often does [child's name] eat meat products such as sausages, frankfurters, devon, ham, hamburgers or chicken nuggets?

Longer list (do not read out): Salami, bacon, meat pies, sausage rolls, luncheon meats, delicatessen meats, meat paste, liver paste, pate, saveloys, cheerios, hot dogs, rissoles, canned meats, smoked chicken, other smoked meats

1.

2. times per week

4. Rarely/never $\quad$ 5. I don't know/can't say

Q5. How often does [child's name] eat hot chips, French fries, wedges or fried potatoes?
1. times per day
times per week
4. Rarely/neve
5. I don't know/can't say
3. times per month
6. Refuse

3. times per month

Q6. How often does [child's name] eat potato crisps or other salty snacks (such as Twisties or corn chips)?
1. Rarely/never
times per week
5. I don't know/can't say
3. $\frac{\text { times per month }}{\text { Refused }}$

Q7. How often does [child's name] have meals or snacks such as burgers, pizza, chicken or chips from places like McDonalds, Hungry Jacks, Pizza Hut, KFC, Red Rooster or local take-away food places?

1.

2.

4. Rarely/never

5. I don't know/can't say

3. $\frac{\text { times per month }}{\text { Refused }}$

Q8. How often does [child's name] have snack foods such as sweet or savoury biscuits, cakes, doughnuts or muesli bars?

$\begin{array}{ll}\text { 1. } \frac{\text { times per day }}{\text { 4. Rarely/never }} & \text { 2. } \frac{\text { times per week }}{\text { I don't know/can't say }}\end{array}$

3. times per month

Q9. How often does [child's name] eat confectionery, such as lollies and chocolate?

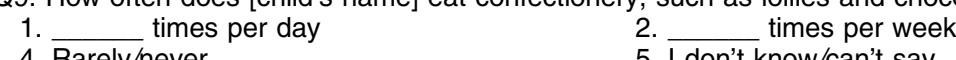

0. How often does [child's name] usually have something for breakfast?
1. Every day
times per week
4. Rarely/neve

don't know/can't say

Q11. How often does [child's name] eat dinner in front of the television?
. Rarely/never
5. I don't know/can't say

3. times per month 


\section{The next few questions are about the quantity of drinks your child usually consumes:}

Q12. How many cups of milk does [child's name] usually drink in a day? Includes cow's milk, soya milk, milk on cereal, flavoured milks (one cup $=250 \mathrm{ml}$, a household tea cup)
cups per day
2. cups per week
3. cups per month
4. Doesn't drink cow's milk or other milk

6. Refused

Q13. What type of milk does [child's name] usually consume?

$\begin{array}{ll}\text { 1. Whole milk (regular, full-cream) } & \text { 2. Low - reduced fat milk }\end{array}$

4. Evaporated or sweetened condensed 5 5. Soya milk, regular. Please specify

. None of the above

$$
\text { 8. Don't know }
$$
3. Skimmed milk
6. Soya milk, reduced fat. Please specify
9. Refused

Q14. How many cups of soft drink, cordials or sports drink, such as lemonade or Gatorade does [child's name] usually drink? $\left(1 \mathrm{cup}=250 \mathrm{ml}\right.$. One can of soft drink $=1 \frac{1}{2}$ cups. One $500 \mathrm{ml}$ bottle of Gatorade $=2$ cups)

1.

4. Doesn't drink soft drink

2. cups per week

5. Don't know

6. Refused cups per month

Q15. How many cups of diet soft drink or diet cordial such as Diet Coke or Diet Sprite or Coke Zero does [child's name] usually drink? ( 1 cup $=250 \mathrm{ml}$. One can of soft drink $=1 \frac{1}{2}$ cups. One $500 \mathrm{ml}$ bottle of Gatorade $=2$ cups)

1. cups per day

2. cups per week

3. cups per month

4. Doesn't drink diet soft drink

5. Don't know

6. Refused

Q16. How many cups of fruit juice does [child's name] usually drink? ( 1 cup $=250 \mathrm{ml}$, a household tea cup or 1 large popper)
cups per day
cups per week
3. cups per month
4. Doesn't drink juice
5. Don't know
6. Refused

Q17. How many cups of water does [child's name] usually drink in a day? ( 1 cup $=250 \mathrm{ml}$, a household tea cup, 1 average bottle of water $=2 \frac{1}{2}$ cups $)$

1. cups per day

4. Doesn't drink water

2. cups per week

3. cups per month 